\section{Verweigerung von vertragsärztlichen Leistungen zugunsten der Privatliquidation}

\section{Einführung}

Das Sozialgericht München urteilte am 23.04.2021, Az. S 28 KA 116/18 über die Klage eines vertragsärztlich tätigen Augenarztes, der sich in dem Verfahren gegen eine Maßnahme eines Disziplinarausschusses einer Kassenärztlichen Vereinigung wehrte. Der Disziplinarausschuss hatte gegen den Augenarzt eine Geldbuße in Höhe von 2.500 Euro verhängt. Hintergrund der Entscheidung war die Erbringung von ärztlichen Leistungen, die der Augenarzt nicht als Vertragsarzt, sondern im Rahmen einer Privatliquidation bei einem gesetzlich krankenversicherten Patienten erbracht hatte. Argumentativ stellte der Augenarzt u. a. darauf ab, dass er aus Kapazitätsgründen keinen weiteren gesetzlich krankenversicherten Patienten an dem Behandlungstag mehr hätte untersuchen und behandeln können und ein medizinischer Notfall nicht vorgelegen habe. Das Sozialgericht bestätigte die Entscheidung des Disziplinarausschusses. Der Sachverhalt ist insofern instruktiv, als dass er verschiedene Aspekte des vertragsärztlichen Pflichtenspektrums in Erinnerung ruft.

\section{Sachverhalt}

Nach einer Behandlung bei einem vertragsärztlich tätigen Augenarzt erhob ein gesetzlich krankenversicherter Patient Beschwerde wegen einer durch diesen Augenarzt vorgenommenen Privatliquidation. Aufgrund einer schmerzhaften Rötung und Schwellung eines Auges hatte der Patient während eines Kuraufenthaltes dessen Augenarztpraxis aufgesucht. In der Praxis war er von einer Empfangsdame gefragt worden, ob er in diesem Quartal bereits bei einem anderen Augenarzt in Behandlung gewesen sei, was der Patient mit Hinweis auf eine regelmäßige Behandlung mit Augentropfen bejahte. Daraufhin wurde ihm mitgeteilt, dass er die Behandlung aus eigener Tasche bezahlen müsse, weil diese nicht mehr bei der Krankenkasse abgerechnet werden könne. Um eine Untersuchung und Behandlung zu erhalten, habe er ein als „Einverständniserklärung“ betiteltes Formular unterzeichnet und 40 Euro bezahlt. In dem Formular erfolgte u. a. der Hinweis darauf, dass der Augenarzt die Leistungen nicht mit einer Krankenkasse abrechnen könne und die Privatrechnung nicht von einer Krankenkasse erstattet würde.

In seiner Anhörung gab der Augenarzt an, dass im Fall des Patienten kein Notfall vorgelegen habe, der eine sofortige Behandlung erfordert hätte. Am Behandlungstag seien 25 Termine vergeben gewesen, so dass im Normalfall keine weiteren Patienten außer absolute Notfälle hätten angenommen werden können. Für nicht dringliche Notfälle bestünden genügend Alternativen vor Ort, sowie gegebenenfalls den augenärztlichen Notfalldienst. Deshalb böte er, insbesondere wenn der Patient im laufenden Quartal schon in vertragsaugenärztlicher Behandlung gewesen sei, an, die Behandlung zunächst als „Zweitmeinung“ zu führen. Das Angebot habe der Patient angenommen, niemand habe ihn dazu gezwungen. Die Alternativen seien genannt worden. Im Rahmen der gewünschten Privatbehandlung habe sich dann eine zusätzliche kurative Komponente herausgestellt, so dass daneben eine vertragsärztliche Behandlung erfolgt sei, die zur Ausstellung eines Kassenrezepts geführt habe. Auf Privatrezept seien keine Medikamente verordnet worden. Er habe den Patienten gründlicher untersucht, als aufgrund der geklagten Beschwerden unabdingbar gewesen wäre, woraus sich dann auch eine zusätzliche Behandlung nach dem Einheitlichen Bewertungsmaßstab ergeben habe.

Die Kassenärztliche Vereinigung empfahl dem Augenarzt, die Voraussetzungen einer Privatliquidation nach $\S 18$ Absatz 8 Bundesmantelvertrag-Ärzte (BMV-Ä) zu prüfen. Dessen Regelung lautet in Satz 2:

„Vertragsärzte, die Versicherte zur Inanspruchnahme einer privatärztlichen Versorgung an Stelle der ihnen zustehenden Leistungen der gesetzlichen Krankenversicherung beeinflussen, verstoßen gegen ihre vertragsärztlichen Pflichten."

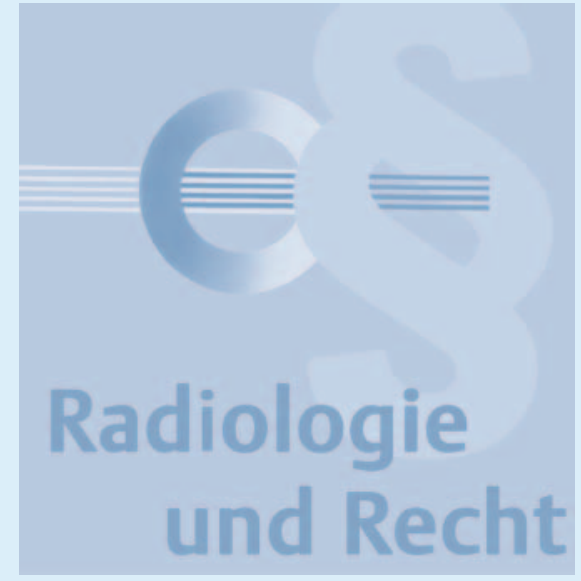

und in Satz 3 in Auszügen:

„Der Vertragsarzt darf von einem Versicherten eine Vergütung nur fordern,

[...]

2. wenn und soweit der Versicherte vor Beginn der Behandlung ausdrücklich verlangt, auf eigene Kosten behandelt zu werden, und dieses dem Vertragsarzt schriftlich bestätigt,

3. wenn für Leistungen, die nicht Bestandteil der vertragsärztlichen Versorgung sind, vorher die schriftliche Zustimmung des Versicherten eingeholt und dieser auf die Pflicht zur Übernahme der Kosten hingewiesen wurde."

Parallel zu dem sozialrechtlichen Verfahren hatte der Patient Zivilklage gegen den Augenarzt erhoben und das Honorar zurückgefordert. Das Amtsgericht wies die Klage ab, so dass der Augenarzt das Honorar nicht zurückzahlen musste.

\section{Wesentliche Begründung des Sozialgerichtes}

Nachdem der Disziplinarausschuss eine Geldbuße in Höhe von 2.500 Euro gegen den Augenarzt festgesetzt hatte, klagte dieser vor dem Sozialgericht gegen die Kassenärztliche Vereinigung. Anders als bei Zulassungs- und Berufungsausschüssen oder Prüfungs- und Beschwerdestellen sind die Disziplinarausschüsse der Kassenärztlichen Vereinigungen keine eigenständigen Behörden. Daraus folgt, dass nicht der Disziplinarausschuss selbst, sondern allein die Kassenärztliche Vereinigung verklagt werden kann, die den Disziplinarausschuss satzungsgemäß eingerichtet hat. 
Das Sozialgericht hatte zunächst die verhängte Maßnahme uneingeschränkt hinsichtlich der Annahme und Feststellung eines schuldhaften Pflichtenverstoßes zu überprüfen. Nach Feststellung eines schuldhaften Pflichtenverstoßes musste das Sozialgericht anschließend prüfen, ob der Disziplinarausschuss bei Auswahl der Maßnahme den ihm eingeräumten Ermessensspielraum in rechtlich zulässigem Maße eingehalten hatte.

Nach dem Sachleistungsprinzip habe der Vertragsarzt seine Leistung als Sachleistung, das heißt für den Kassenpatienten gänzlich kostenfrei zu erbringen. Es könne offenbleiben, ob im Fall des Patienten ein Notfall im Sinne des $§ 76$ Absatz 1 Satz 2 SGB $V$ vorgelegen habe oder nicht. Unabhängig von der Frage der dringenden Behandlungsbedürftigkeit sei der Patient auf jeden Fall behandlungsbedürftig gewesen. Nach Überzeugung des Sozialgerichts traf den Vertragsarzt die Pflicht, den Patienten zu behandeln. Nach $\S 13$ Absatz 7 Satz 3 BMV-Ä dürfe ein Vertragsarzt, sofern kein Ausnahmefall nach den Sätzen 1 und 2 vorliege (Anmerkung: Die elektronische Gesundheitskarte legt der Patient nicht vor und es besteht keine akute Behandlungsbedürftigkeit), die Behandlung eines Versicherten nur in begründeten Fällen ablehnen. Grundsätzlich könne eine kapazitätsmäßige Überlastung des Vertragsarztes einen derartigen begründeten Ablehnungsgrund darstellen. An dem Behandlungstag habe eine solche Überlastung beim Augenarzt jedoch nicht vorgelegen. Andernfalls hätte der Augenarzt keine Zeit gehabt, bei dem Patienten an diesem Tag eine ausführliche, privatärztliche Behandlung inkl. kleinchirurgischen Eingriffs vorzunehmen. Indem der Augenarzt die begehrte GKVBehandlung als privatärztliche Behandlung anbot und abrechnete, verstieß er gegen das Sachleistungsprinzip sowie gegen die Vorschrift des $\S 128$ Absatz 5a SGB V, der lautet:

„Vertragsärzte, die unzulässige Zuwendungen fordern oder annehmen oder Versicherte zur Inanspruchnahme einer privatärztlichen Versorgung anstelle der ihnen zustehenden Leistung der gesetzlichen Krankenversicherung beeinflussen, verstoßen gegen ihre vertragsärztlichen Pflichten. “
Einer grundsätzlichen Vertragsfreiheit des Vertragsarztes stehe $\S 13$ Absatz 7 BMV-Ä entgegen. Über diese Behandlungspflicht habe der Augenarzt den Patienten fehlerhaft informiert und somit unzulässig zur Unterzeichnung der Einverständniserklärung und Zahlung des Honorars „motiviert“. Schließlich widerspreche die gegenüber der Kassenärztlichen Vereinigung erfolgte Abrechnung der Aussage der privatärztlichen „Einverständniserklärung“, wonach die vereinbarten Leistungen nicht mit der Krankenkasse der Versicherten abgerechnet werden könnten. Dementsprechend, so das Sozialgericht, liegen die Voraussetzungen des $\oint 18$ Absatz 8 Satz 3 Nr. 2 BMV-Ä nicht vor. Indem der Augenarzt den Patienten fehlerhaft über seine - tatsächlich bestehende - Behandlungspflicht sowie die Voraussetzungen und Rechtmäßigkeit einer Privatliquidation aufklärte und die (mögliche) Doppelabrechnung bei der Kassenärztlichen Vereinigung verschwieg, habe der Augenarzt den Patienten auf unzulässige Weise dazu beeinflusst, das schriftliche Einverständnis zur Zahlung des ärztlichen Honorars zu geben.

Nachdem das Sozialgericht den vorbeschriebenen Pflichtenverstoß festgestellt hatte, war über die Ermessensausübung hinsichtlich der Auswahl der Maßnahme und deren Höhe zu entscheiden. Der Augenarzt habe schuldhaft gehandelt und zumindest grob fahrlässig gegen das Sachleistungsprinzip verstoßen sowie zumindest grob fahrlässig eine unzulässige Doppelabrechnung vorgenommen. Ihm hätte bewusst sein müssen, dass die Abrechnung der Leistung sowohl als Privatleistung als auch über die Krankenversichertenkarte nicht rechtmäßig ist.

Nach Auffassung des Sozialgerichts habe die Kassenärztliche Vereinigung zutreffend ausgeführt, dass der Augenarzt mit der Nichtübernahme der Behandlung sowie der Nichtbeachtung des Sachleistungsprinzips gegen eine elementare vertragsärztliche Pflicht verstoßen hat. Trotz mehrfacher Aufforderungen durch die Kassenärztliche Vereinigung habe er sich geweigert, das Honorar an den Patienten zurückzuzahlen und damit fehlende Einsicht in sein pflichtwidriges Verhalten gezeigt. Erschwerend sei zu berücksichtigen gewesen, dass der Augenarzt bereits disziplinarrechtlich in
Erscheinung getreten sei und es sich bereits um dessen zweiten Verstoß - der erste Verstoß fand im Jahr 2012 statt, der gegenständliche erfolgte im Jahr 2015 - gegen das Sachleistungsprinzip handele.

\section{Exkurs - Unzulässigkeit der teilweisen Abrechnung von vertragsärztlichen Leistungen}

Der Augenarzt hatte u. a. in seinen Stellungnahmen angeführt, dass er Leistungen erbracht hätte, die nicht in der vertragsärztlichen Versorgung erbracht werden könnten. Solche oftmals eher teureren oder aufwändigeren Leistungen finden sich auch in der Radiologie. Dies führt zu dem Problem, dass nicht jeder Selbstzahler in der Lage oder dazu gewillt ist, das nach der Gebührenordnung zu verlangende Honorar zu entrichten. In solchen Fällen stellt es für einen Vertragsarzt oder ermächtigten Arzt keine Lösung dar, eine vertragsärztliche Leistung mit einer Gebührenordnungsposition nach dem Einheitlichen Bewertungsmaßstab anzusetzen, wenn die tatsächlich erbrachte Leistung im Umfang über den der Gebührenordnungsposition hinausgeht. Zuletzt hatte das Bundessozialgericht in seinem Beschluss vom 11.03.2021, Az. B 6 KA 41/20 B festgestellt, dass nicht nur derjenige Vertragsarzt gegen die Pflicht zur peinlich genauen Abrechnung verstößt, der nicht erbrachte Leistungen abrechnet, sondern auch derjenige, der tatsächlich erbrachte Leistungen nicht oder nicht vollständig abrechnet. Daher bleibt beispielsweise eine CT-gesteuerte schmerztherapeutische Intervention eine CT-gesteuerte schmerztherapeutische Intervention und kann nicht als CT-Untersuchung von Teilen der Wirbelsäule abgerechnet werden.

\section{Fazit}

Die Entscheidung zeigt einmal mehr auf, wie schmal der Grat zwischen zulässiger Privatliquidation eines Vertragsarztes oder ermächtigten Arztes und einem Verstoß gegen die vertragsärztlichen Regelungen bei gesetzlich krankenversicherten Patienten ist. Aufgrund der unverändert bei den Radiologen, Nuklearmedizinern und seit dem ersten Quartal 2021 auch bei den Strahlentherapeuten bestehenden Honorarbudgetierung gibt es zahlreiche legale 
und illegale Lösungsversuche, den sinkenden Honoraren bei steigenden Kosten und Anforderungen wenigstens ausgleichend zu begegnen. Ein Dilemma dieser Entwicklung zeigt der Fall des Augenarztes anschaulich: da er grundsätzlich für eine Untersuchung und Behandlung bereitstand, konnte er nicht die Behandlung des gesetzlich krankenversicherten Patienten zulasten der Krankenversicherung ablehnen. Gleichzeitig sieht sich ein Vertragsarzt dem Risiko des Überschreitens der Plausibilitätszeiten ausgesetzt, wenn er stets und zu jeder Zeit jeden Patienten annehmen und untersuchen oder behandeln würde. Eine vergleichbare Situation ergibt sich daneben, wenn sich ein Vertragsarzt in seiner Praxis vertreten lässt, weil er sich vermeintlich - im Urlaub befindet, aber tatsächlich nur in seiner GKV-Praxis im Urlaub ist, für Selbstzahler und Privatpatienten dagegen uneingeschränkt in der Praxis zur Verfügung steht. Einen versicherungsartabhängigen Urlaub gibt es im Vertragsarztrecht nicht; entweder befindet sich der Vertragsarzt im Urlaub oder nicht. Ein in dieser Weise handelnder Vertragsarzt verstößt ebenfalls gegen die vertragsärztlichen Pflichten, sodass Disziplinierungsmaßnahmen seitens der zuständigen Kassenärztlichen Vereinigung drohen.
Immer wieder ist es unangenehm festzustellen, dass im Disziplinarrecht die Faktoren, die für die Festlegung der Intensität einer Maßnahme herangezogen werden, grundlegend fragwürdig sind. So zogen der Disziplinarausschuss und später das Sozialgericht den für den Vertragsarzt nachteiligen Schluss, dass er sich nicht zur Rückerstattung des Honorars bereit erklärt habe und damit wenig Einsicht zeige. Dem vorausgegangen waren mehrere Aufforderungen der Kassenärztlichen Vereinigung an den Vertragsarzt, das Honorar zurückzuzahlen. Dabei hatte der gesetzlich krankenversicherte Patient vor einem Amtsgericht die Klage auf Rückforderung des geleisteten Honorars rechtskräftig verloren. Der Vertragsarzt hatte daher keine zivilrechtliche Verpflichtung, dass erhaltene Honorar zurückzuzahlen. Der Disziplinarausschuss nahm, sprach dies aber nicht offen aus, eine moralische Pflicht zur Rückzahlung an. Eine moralische Pflicht korreliert dabei aber mehr mit einer bestimmten Erwartungs- oder Forderungshaltung des Disziplinarausschusses und entspricht jedenfalls hier gerade keiner rechtlichen Verpflichtung. Zwar stellte das Sozialgericht fest, dass die Entscheidung des Amtsgerichtes nur zwischen dem Patienten und dem Vertragsarzt ergangen war und die Kassenärzt- liche Vereinigung nicht an dem Verfahren beteiligt war, so dass die Kassenärztliche Vereinigung und damit der Disziplinarausschuss nicht an das Urteil gebunden war. Die Grundlage einer gesetzlichen Verpflichtung zur Rückerstattung legten aber weder der Disziplinarausschuss noch das Sozialgericht in seiner Urteilsbegründung dar. In der Spruchpraxis der Disziplinarausschüsse finden sich häufig entsprechende für den Vertragsarzt nachteilige Feststellungen, die auf der Annahme von moralisch anstößigen und je nach persönlichem Empfinden durchaus kritikwürdigen Verhalten beruhen, denen es aber dennoch an einer gesetzlichen Grundlage fehlt. In diesen Fällen erscheint das Disziplinarrecht merkwürdig abgekoppelt und aus der Zeit gefallen zu sein.

René T. Steinhäuser

Rechtsanwalt

Rechtsanwälte Wigge

Großer Burstah 42

20457 Hamburg

Telefon: (040) 3398 705-90

Telefax: (040) 3398 705-99

Internet: www.ra-wigge.de

E-Mail: kanzlei@ra-wigge.de 